\title{
Aeronautics design and manufacture integrated enterprise model
}

\author{
Likang Song ${ }^{1}$, Xiumao Yang ${ }^{1}$, Chao $\mathrm{Fu}^{2}$, and Jihong Liu ${ }^{2, *}$ \\ ${ }^{1}$ Hongdu Group Company of Aeronautical Industry, 330024 Nanchang, China \\ ${ }^{2}$ School of Mechanical Engineering and Automation, Beihang Uiversity, 100191 Beijing, China
}

\begin{abstract}
In order to realize the integration and concurrent of design and manufacture, enhance the efficiency of aircraft development enterprises, a model centre based design and manufacturing integrated enterprise model is proposed. The model centre consists of central system models, product models and process model. The design and manufacture integrated development model based on the unified model centre is discussed. Finally, a maturity control method supporting design and manufacturing integration is proposed.
\end{abstract}

\section{INTRODUCTION}

Aviation product development is a comprehensive technology[1]. The design process of aircraft is a comprehensive trade-off and optimization of various systems and technologies. Practice has proved that the integrated design and manufacture of aircraft is an important technical to improve product quality and reduce product development cycle [2]. The core idea of design and manufacture integrated is the application of concurrent engineering, digital engineering and collaborative design technology etc. to realize seamless integration and parallel development of aircraft design and manufacture.

Aircraft development is complex system engineering. The enormous challenge for aeronautical manufacturing is to achieve the best in terms of performance, cost and planning cycle in aircraft development [3]. Modern aircraft design and manufacturing technology based on digitalization is changing the traditional way of aircraft development. Aircraft design and manufacturing technology from the digital three-dimensional design and manufacturing to the process as the centre of the integrated development model transition. A variety of individual digital technology, network technology, advanced manufacturing technology are gradually applied to the aircraft development process, and in the digital definition, product data management, information integration and other aspects of significant progress. However, the current aircraft development enterprises are faced with the following problems: cannot achieve the "demand - functional logic model - behaviour model - physical model" of a single data source management and association, trace management; cannot be established by the demand, functional logic model, behaviour model, physical model Constitute the BOM tree, cannot implement the model of the data baseline / version management and configuration technology state management; does not support the performance of the prototype (FDMU) in the ring design. So, a model centre based design and manufacturing integrated enterprise model is proposed. The model centre consists of central system models, product models and process model. The design and manufacture integrated development model based on the unified model centre is discussed. Finally, a maturity control method supporting design and manufacturing integration is proposed.

\section{Model centre of designing and manufacturing integrated development enterprise}

Model is the key and core of aircraft design and manufacture integrated enterprise. As shown in Figure 1, model centre that support the aircraft design and manufacture integration development include the central system model, product model, process model and knowledge model. Among them, the central system model is a special product lifecycle model. Knowledge model is divided into product knowledge model and process knowledge model, and it has no fixed form of expression. Integrated design and manufacture of aircraft is based on the system engineering framework, through the construction of enterprise products in the whole life cycle of product model, process model and knowledge model, and on this basis to carry out the corresponding engineering design, manufacture, and service.

\subsection{Central system model}

Corresponding author: ryukeiko@buaa.edu.cn 
The central system model is the primary work of system development. Unified central system model that defines system requirements and establishes traceability relationships between requirements and design at all levels. With the help of standard software environment and data exchange, the model of product model and process model in each stage of product lifecycle are integrated, which can achieve design, complex product manufacturing, services, scrap etc. under a single data source. The central system model first emphasizes the comprehensiveness, accuracy and consistency of the system description. By associating the related parameters of each model in the product life cycle and each stage, the specialized engineers of various disciplines and the subsequent technical and service personnel can work on the basis of the system architecture model. The data is taken from a common database to design, analyse and optimize using the discipline's model and software tools. That is to say, under the MBSE idea, the real single data source technology is based on the central system model.

\subsection{Product model}

Product is the main output of manufacturing enterprises. The product model mainly completes the functions stipulated in the application framework according to the requirements of the business framework, MBD model is the main form of product model. The purpose of MBD is to annotate $3 \mathrm{D}$ models with engineering information on traditional 2D drawings. Those kinds of annotated information descript what the product is and how it should be manufactured. However, currently the supportive information behind the design results (design knowledge) has not been added into models. This sort of supportive information for design explains how designers came up with the design results, while most of them are preserved in design instruction booklet and are rarely used. So to integrate design process information into 3D models can assist designers to understand the product design and reuse them in new products. That is also an effective approach to preserve knowledge, which is precious for an enterprise, and make them more helpful.

MBD data sets can be divided into geometric model, design information, simulation analysis information, and manufacturing information. Geometric model includes $3 \mathrm{D}$ entity and auxiliary geometry of the product; Design information includes product size, tolerances, assembly relations, material selection, technical requirements, and parts list; manufacturing information includes the process planning and assembly sequence of parts processing; Simulation analysis information includes finite element simulation, assembly process simulation and behaviour simulation.

\subsection{Process model}

The core value chain of modern enterprise is the business process. The process model is the description and coordinator of the relevant resources, information, tools and other elements in the enterprise to execute any process, support enterprises to change their products from concept to material and then to use. In general, the process model of enterprises can be divided into two categories: engineering process model and management process model.

The engineering process model is abstracted through engineering practice. Especially the development of complex products, such as aerospace, is an interdisciplinary complex system engineering oriented to the whole life cycle. A detailed analysis of the activities in various stages is required to describe the business process. While, the management process model aims to realize agile, refine and lean management. Through explicit, structured, and standardized business process, we can achieve the modelling, simulation and evaluation of enterprise management business processes and the implementation, monitoring, interaction and control of subsequent application processes. By modelling and understanding the behaviour of enterprise/system, through the simulation study and prediction of the future dynamics of the enterprise system, it provides a basis for the final construction of a new enterprise management method and mode.

\section{The design and manufacture integrated development model based on the unified model centre}

\subsection{Model-based system engineering product development}

Combining MBSE model based system engineering methodology, this paper aimed at the development characteristics of aircraft system development and the development of aircraft design and manufacturing, studied the model based system engineering product development system. Through the construction of products covering the whole life cycle of information technology system architecture, the business flow and data flow in the process of product development are realized, and the model-based demand engineering, model-based design engineering, model-based test (flight test) engineering, model based manufacturing engineering, and system life cycle management are developed for the product MBSE.

Through the model to support the requirements of the definition, design, manufacture, integration and validation. With the application of model - based system engineering, the model based on SysML is introduced in the conceptual stage of product development to modle the requirements definition, function analysis and architecture design. The correctness and completeness of requirements and functions are validated in advance by model execution. The model execution ensure collaboration between systems, and collaboration within each component of the system meets requirements. After the function logic of the system is modeled and verified, the function analysis loop is implemented. Through the establishment of a formal system engineering model to describe detailed definitions and system functions and 
control logic of the requirements of each stage of the aircraft development, this turn ways and experience of product development into structured knowledge; and with the system engineering model as the core, the digital expression model of different disciplines is integrated to achieve multidisciplinary collaboration; at the same time, using CAE technology to achieve simulation driven products, components and parts design, and from the point of view of system engineering, from demand management, functional analysis, simulation and virtual design simulation drive. On the other hand, virtual experiment is used to realize parts virtual test, component virtual structure and product simulation, and realize virtual testing, virtual synthesis and virtual verification of requirements, functions and design. And with the function (performance) virtual prototype bridge, the establishment of functional decomposition and virtual verification of the association to the implementation of the system engineering model to drive the model development plan and process execution, and ultimately the formation of double "V" system engineering development model. Through top-down, demand driven system development model, improve the process, methods and means to promote the efficiency of system development.

3.2 Design and manufacture integrated development based on Model Center

Design and manufacture integrated development enterprise based on model center is through the establishment of a single data source model center, multi-disciplinary, cross-sectorial product design, manufacturing and management, a fundamental reduction in product design, manufacturing and maintenance support time and cost. Its fundamental feature is based on the model, the core of knowledge as the driving force, for the whole life cycle of the system for business integration, data integration, parallel collaboration, agile and efficient product development with the guidance and support of the system engineering and knowledge engineering.

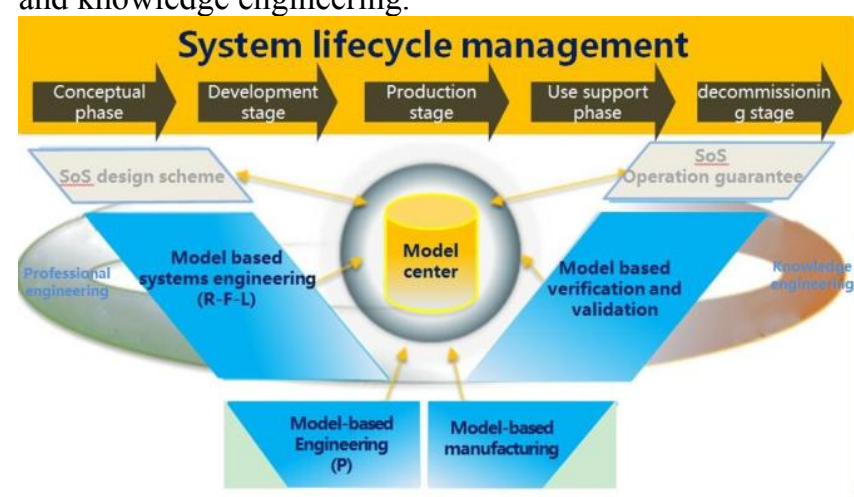

Figure 2. Enterprise development model based on unified model centre

As shown, in order to effectively use between various disciplines and the professional advantages, based on the model design and manufacturing integration in the centre of the research and development process is divided into different stages. And each stage needs to fully take advantage of the existing research, and use different disciplines software for the corresponding development activities.

Model based system engineering

In the conceptual phase, the requirements of the product are captured to define the operational parameters and performance that need to be satisfied during the product development process and form an entry document of requirements. In the process of functional analysis and architecture design, we can implement the analysis and refinement process from black box to white box, from product to subsystem and the formation of product design through the decomposition of requirements. And through the operation of the design scheme, the logical operation of the design scheme is completed. In this process, the SysML language can be used to analyse the product refinement process by the system engineering model method. Finally, the correctness of the logic in the design process is verified by the state diagram [4].

The results of the analysis are simulated by multi physics when using MBSE thought to analyse the design process of product. During the design process, the problems that may arise in the process of product development are understood and solved from the root. By establishing the product model and integrating it with the Modelica simulation software, the performance simulation of the product is realized, and the multi physical simulation in the design process is realized. As shown in Figure 3, through the data integration of DOORS, Rhapsody, SimulationX and VPM/CATIA, we can establish the BOM structure of R-F-L-P, which is composed of requirements, function logic model, behaviour model and physical model. This is used to support enhanced design capability by digital prototyping (DMU) to performance prototype (FDMU) design.

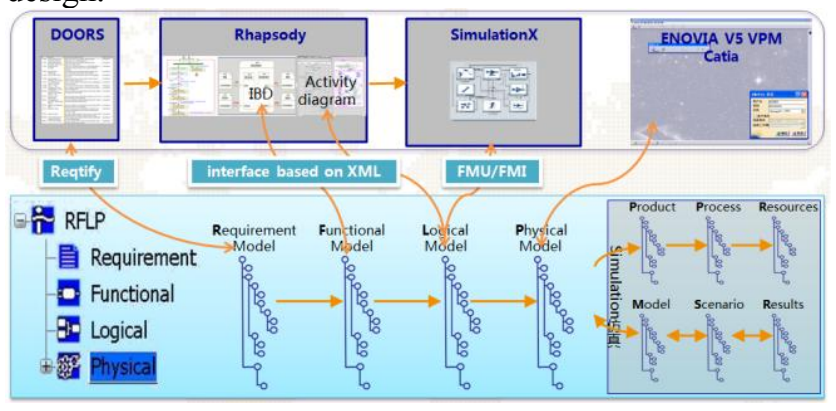

Figure 3 MBSE Integration model

- Model-based engineering

Model-based engineering includes digital collaborative design and model-based analysis. Digital collaborative design is a digital optimization system with annotated 3D models at the core. Its purpose is by defining products via annotating models in the upstream of PLM to ensure the smooth progress of works in downstream. [5,6]. Model based analysis takes the PDM system as the platform; it integrates the $3 \mathrm{D}$ design tools and simulation analysis tools to build a proper design and analysis environment. This is in order to achieve unified management design model and analysis of data 
and its version. Model based simulation uses the model to be divided into three categories: equipment model, cognitive model and message model. Model based simulation technology is based on the computer; take the principle of similarity, information technology and other related applications in the field of basic theory and technology as the foundation [7].

\section{- Model - based manufacturing}

Model based manufacturing includes model-based process design and model-based manufacturing execution [8]. Model based process planning is based on product design results, and the process planning of product manufacturing process is the direct basis of product manufacturing process. The process is based on CAPP software, including design and Simulation of 3D parts process, design and simulation of 3D assembly process, model based tooling design and management, model based process resource management and so on. Model based manufacturing is the development of modern manufacturing technology to meet the increasingly diversified product requirements, and the expression and application of product design and manufacturing data is the core issue of digital manufacturing.

\section{- Model-based validation and delivery}

Model based recognition and delivery is the digital model of existing design results that are presented to stakeholders in a visual form [9]. This avoids the delivery of the physical prototype, helping to shorten the entire life cycle of the product and accelerate the development of the product. This process uses the twinmodel idea to digitize and virtualize the actual product and show the whole structure of the product.

\section{Maturity control technology for design and manufacture integrated development}

The model center solves the problem of uniform data source for collaborative processes of design and manufacture, and maturity control is the basic standard for concurrent of design and manufacture.

At present, there are many kinds of technology status control methods based on their own standards, such as stage, milestone, baseline and so on. Therefore, a model maturity management mechanism with 16 levels of maturity is proposed, which provides the necessary technical support for the concurrent collaborative design of design, fixture, and quality control.

For aircraft development projects and design and manufacturing departments who expects to realize concurrent and collaborative development of aircraft,Only according to the five stages of aircraft development to determine the organization, planning and management of the project must be too extensive. In order to shorten the development cycle of the aircraft and improve the development efficiency, the maturity model of product covering the whole life cycle of aircraft is proposed to explore collaborative work patterns and work content of the design and manufacturing department in different maturity level under the organizational model.

The process of the development of the aircraft project, from the start of the project to decommissioning, is divided into 16 Level maturity model based on major events and major assessment point of view. Fig 1 shows the maturity level of 16 . ML0 start of the project, ML1 formal initiation of the project, ML2 Technical description of aircraft design, ML3 Determination Of preliminary overall technological scheme, ML4 Basic terminology, ML6 start of parts/fixture manufacturing, ML8 complete assembly of the fixture,ML9 01 plane into the final assembly, ML10 01 plane enter the test station, ML11 01 plane maiden flight, ML12 identification of flight test, ML15 halt production, ML16 decommissioning, also including ML5 appraisal of design scheme, ML7 detailed design examination, ML13 review of design finalization, ML14 review of production finalization and so on.

The product maturity model of the aircraft's full life cycle is established. In order to achieve the interdisciplinary concurrent collaborative development between the design, manufacturing and other professional departments, it is necessary to clarify the work content of each professional department under each maturity level. Based on the unified product life cycle model with maturity level, through the development of the corresponding standards, criterion and management system, it is necessary to combine with the actual situation of enterprises to achieve concurrent development in the final product design, process/fixture design and manufacturing. It becomes more efficient relying on PLM software platform.

\section{Conlusion}

As the main body of modern aircraft development, design and manufacturing integration is not only a technical problem, but also a social and technical problem involving many aspects, such as enterprise organization, business process, development strategy, planning and so on. This paper summarizes the model centers of $\mathrm{R} \& \mathrm{D}$ integrated manufacturing enterprises, including central system model, product model and process model. On this basis, the design and manufacturing integration development model using the unified model center is discussed, and the maturity control technology for concurrent design and manufacture is also introduced. Research on aircraft design and manufacturing integration enterprise model can guide the transformation of aircraft manufacturing enterprises and ensure the effectiveness of the development of design and manufacturing integration technology.

\section{Acknowledgements}

This work has been supported by Project of National Key Technology R\&D Program through approval No. 2015BAF17B01. 


\section{References}

1. Selvaraj, P., P. Radhakrishnan, and M. Adithan. An integrated approach to design for manufacturing and assembly based on reduction of product development time and cost, The International Journal of Advanced Manufacturing Technology, 42(1): 13-29 (2009).

2. Ulrich, Karl T., and David J. Ellison. Internalization and Integration of Design and Production. Production and Operations Management, 14(3): 315-330(2005).

3. Su, Daizhong, and Nariman Amin. A CGI-based approach for remotely executing a large program for integration of design and manufacture over the Internet. International Journal of Computer Integrated Manufacturing, 14(1): 55-65(2001).

4. Kaslow, David, et al. Integrated model-based systems engineering (MBSE) applied to the Simulation of a CubeSat mission. Aerospace Conference, 2014 IEEE.
5. Graignic, Pascal, et al. Complex system simulation: proposition of a MBSE framework for designanalysis integration. Procedia Computer Science, 16(1): 59-68(2013).

6. Alemanni, M., F. Destefanis, and E. Vezzetti. Model-based definition design in the product lifecycle management scenario. The International Journal of Advanced Manufacturing Technology, 52(1): 1-14(2011).

7. Li C, Wong W H. Model-based analysis of oligonucleotide arrays: expression index computation and outlier detection. Proceedings of the National Academy of Sciences, 98(1): 3136(2001).

8. Butterfield, J., et al. Optimization of aircraft fuselage assembly process using digital manufacturing. Journal of Computing and Information Science in Engineering, 7(3): 269275(2007).

9. Estefan, Jeff A. Survey of model-based systems engineering (MBSE) methodologies. Incose MBSE Focus Group 25(8) (2007).

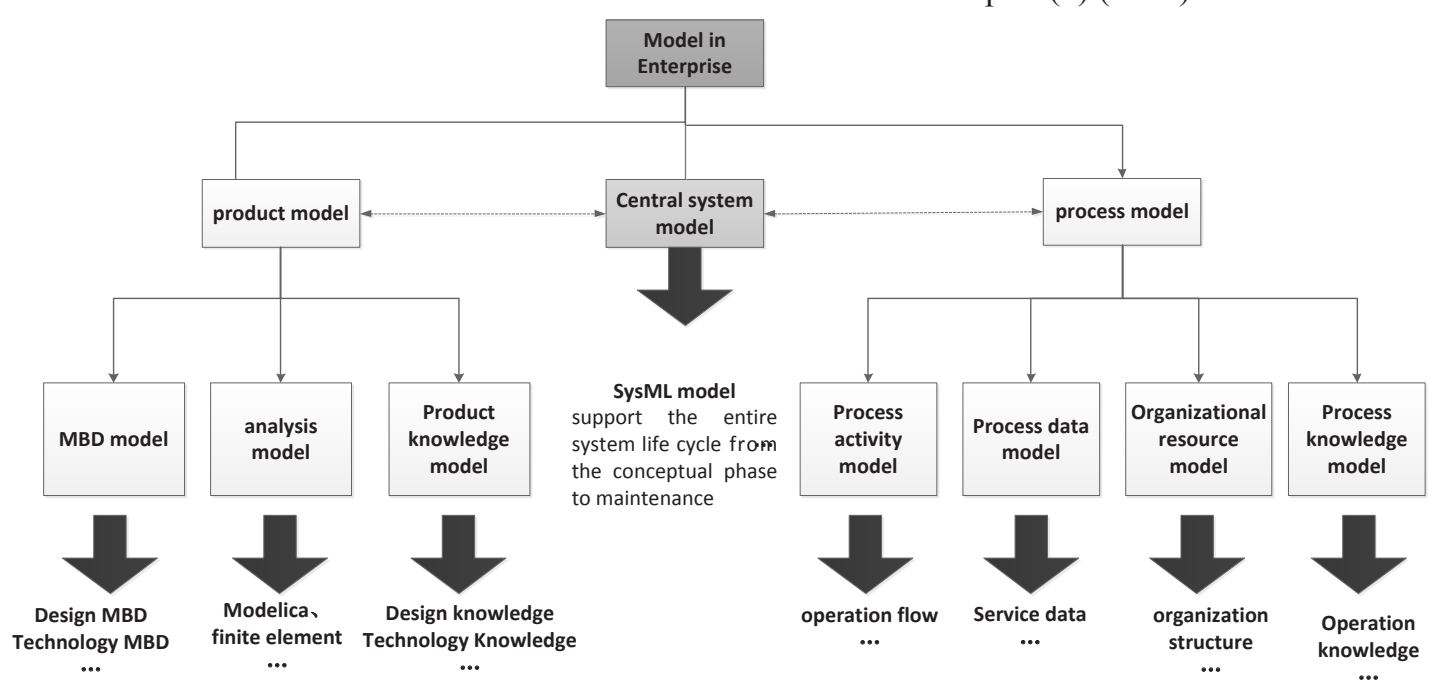

Figure 1. Model centre of designing and manufacturing integrated development enterprise

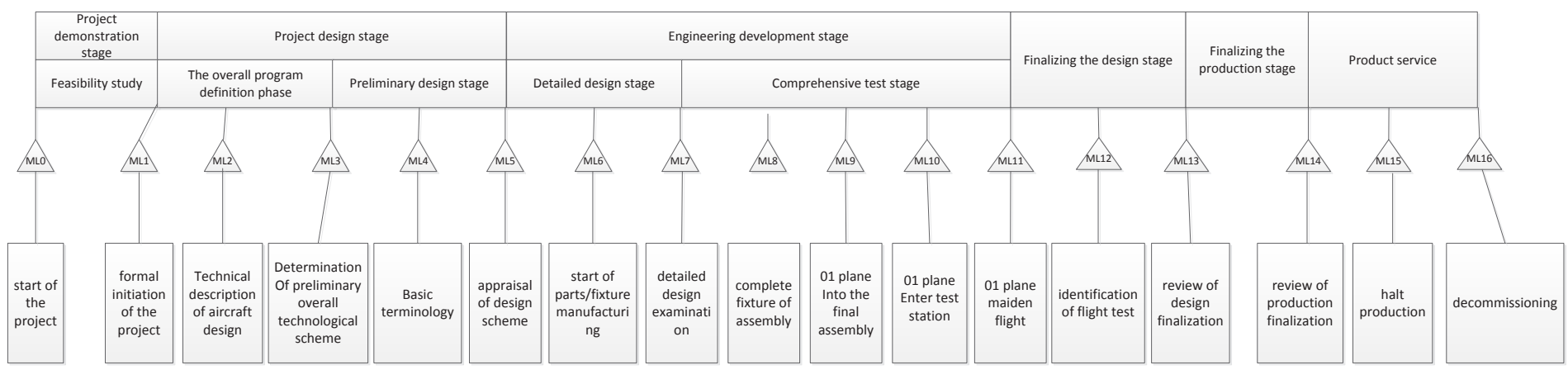

Figure 4. Product maturity model 